\title{
A novel adaptive tolerance filter for random valued impulse noise suppression in digital grey and color images
}

\author{
Geeta Hanji $^{1 *}$, M. V. Latte ${ }^{2}$ \\ ${ }^{I}$ PDA Engg. College, Gulbarga, Karnataka, India \\ 2 JSS Institute for Technical Education, Bangalore, Karnataka, India \\ *Corresponding author E-mail: geetanjalipatil123@gmail.com
}

Copyright (C) 2015 Geeta Hanji, M.V.Latte. This is an open access article distributed under the Creative Commons Attribution License, which permits unrestricted use, distribution, and reproduction in any medium, provided the original work is properly cited.

\begin{abstract}
During acquisition, transmission, storage and retrieval, quite often digital images become noised and mask the important details of an image and also present themselves with an ugly look. Such images if not processed properly, become useless for subsequent image processing operations like segmentation, classification etc. Hence the quality improvement (through denoising) of such noisy images becomes an important operation in the field of image processing. This paper proposes a two stage detection-estimation based filtering system to suppress random valued impulse noise from digital grey and color images based on neighborhood statistics of pixels of the working window under consideration. An adaptive working window and the adaptive tolerance computed from the pixels belonging to the current filtering window facilitates an appropriate detection of noisy pixels in the first phase, followed by the simple and computationally efficient restoration strategy in the second stage. The Simulation results prove that the proposed scheme exhibits much superior performance in comparison with other state of art image filtering methods in suppressing the random valued impulse noise from the digital grey and color images.
\end{abstract}

Keywords: Adaptive Tolerance; Denoising; Detection-Estimation Based Strategy; Neighborhood Statistics; Random Valued Impulse Noise (RVIN); Peak-Signal-To-Noise Ratio (PSNR); Mean Square Error (MSE); Structural Similarity (SSIM) Index.

\section{Introduction}

Noise suppression from the images with detail preservation such as edges and the other vital features is one of the major challenges for the researchers in the field of image processing [1]. Noise suppression via image denoising is an important sub domain of digital image processing field and essentially involves the subjective and objective quality improvement of the degraded and noisy digital images based on the prior knowledge of the mathematical or probabilistic model of image degradation [1]. One of the commonly interfering noises in digital images is impulsive noise that may be added with the images during acquisition, storage, transmission and retrieval. The interference of noise in images might affect the results for further processing, such as edge detection, image segmentation, data compression, object recognition and classification [1], [2].Image denoising is regarded as an essential task in digital image processing and should be performed on the contaminated images before any other image processing operations [3]. The challenge of this task is how to denoise while keeping the important image details. Two types of impulse noise are 'fixed valued' (or salt and pepper) or 'random valued'. In case of salt and pepper noise the noise affected pixels assume the maximum [or ' $255^{\text {'] }}$ or minimum [or' 0'] gray levels, whereas in case of random valued impulse noise the noisy pixels may be any value within the dynamic range of minimum or maximum (i.e. ' 0 ' to ' 255 ' range) gray levels [3], [4].The prime objective of denoising filters is to suppress the image noise while preserving image details such as edges and other vital features in the restored images. There are plenty of works proposed in the literature to meet these requirements [3], [4]. A detailed survey of image denoising methods is available in [2], [3], [4] and [5].

Two basic approaches to image denoising are 'spatial' and 'frequency domain' methods. A traditional way to remove noise from the images is to employ the spatial filters which are further classified into non-linear and linear filters. Since past several years linear filters have been the dominating filter class only because of the sound theoretical basis 
provided by the theory of linear systems and a very good computational efficiency. Despite the strong linear system theory, it is generally observed that not all signal / image processing problems can be satisfactorily solved by them [3], [4]. It is because linear filters tend to blur sharp edges, fail to remove heavy amount of noise effectively and their performance is still poor in the presence of signal dependent noise. In contrary to this, with non-linear filters the noise is removed without any attempts to explicitly identifying it. A popular class of non-linear filter is median filter [1], [3] and has been proved very useful in denoising the fixed value impulsive type of noise with detail preservation capability. An 3important limitation of the median filter is that the filter output is always constrained, by definition, to be one of the samples in the input window. In general it is studied that the median filter loses nearly $40 \%$ efficiency over the sample mean when used as a noise estimator in non-impulsive noisy environments [3], [4]. Several developments over median filter were carried out and many median based filters were proposed such as Standard Median Filter (SMF) [4], Adaptive Median Filter (AMF)[5] and Progressive Switching Median (PSM) filter[6] etc. Moreover many nonlinear filters which are based on order statistics or ranking are enlisted as Minimum, Median, Maximum, Midpoint and Alpha trimmed filters [4], [5].These filters exhibit better results only for low density noise removal but presents blurring for high noise density level. Each filter has its own advantages and disadvantages. It is also observed that the performance of median filter and several variants are less promising when applied to the images noised with random valued impulsive noise [3], [4] and [5].

In the case of the Decision Based and Switching based Median Filters (DBSMF) [7], the decision regarding detection of noisy pixel is difficult. Additionally the details and edges are not recovered satisfactorily especially for high level noise density. Moreover decision based filters even though replaces neighborhood pixel for fixed impulse noises, they introduce streaking effects.

Another filter based on decision approach, called Decision Based Unsymmetrical Trimmed Median Filter (DBUTMF) [8] in which median value is replaced after trimming extreme values on both sides, so that the pixels with luminance values ' 0 ' and ' 255 ' are removed by trimming process itself. The limitation here is that though this approach reduces the computational complexity, it is difficult to design the trimming measure since it is unsymmetrical in nature and the performance results are poor for the high noise density.

To overcome the above problem, symmetrical trimming approach was proposed where trimming is performed at both ends by exploiting Alpha Trimmed Mean (ATM) [9] filter. Disadvantage of this approach is that it not only trims corrupted pixels but often trims uncorrupted pixels too which in turn leads to loss of important image details and consequences with blurring effect.

In view of the above drawback, a novel work on An Iterative Truncated Arithmetic Mean Filter and its Properties [10] is presented to overcome the limitations of the trimming approaches to yield better denoising performance. Another method suggested as a remedy in this context is an Unsymmetrical Trimmed Median Filter (UTMF) [11].This method sorts all the elements in ascending or descending order, and in that order the noisy pixels are removed first phase. In the second phase the remaining pixels alone are considered for computing median value and the same is utilized for the replacement of centre pixel of the selected window under consideration.

The key drawback of switching median filters is that these filters are non-adaptive to noise density variation and wrong classifying pixel behavior at high density noise level [6], [7]. The alternate solution for this method is proposed by Srinivasan and Ebenzer [13]. In the first phase, adaptive median filter is exploited to classify the nature of pixel and in the second phase special regularization method is employed only for noisy pixels in order to preserve the edges effectively. Demerit of this two phase algorithm is the processing time which is more due to selection of very large size window of range 39x39 for both phases to get optimum result. It has an added drawback of more complex circuitry and difficulty for the determination of smoothing factor $\beta$.

An efficient approach by How-Lung presents the Noise Adaptive Soft Switching Median Filter (NASSMF) [14] for detection and filtering. In this technique small window size is chosen for low density noise level and big window size is selected for high density noise level. In addition to this greater weight assigned for closer pixels and lesser weights for far pixels intensity values.

To overcome the above problem of complexity, new replacement techniques have been implemented instead of conventional median value replacement for corrupted pixel [15], [16], [17], [18].These approaches exploit the neighborhood pixels information for replacement unlike AMF and other filters which uses only median value. Specifically at the higher level noise density, there are situations that the median value itself might be noisy value even after applying filtering. In that case the centre pixel is replaced with the processed immediate neighborhood pixel [19], hence edge could be preserved which in turn reduces computational complexity.

The benefits such as reduction in computational complexity, hardware complexity and increase in speed of operation etc are possible to achieve using several methods of sorting [11], [12], and [20]. It has been suggested by the Rajamani et al., and is known as Lone Diagonal Sorting (LDS) [12] algorithm in which three right diagonal pixels were considered for sorting and median value of three elements is computed instead of nine elements sorting as in conventional approach. Thereby decrease in number of sorting and computational complexity.

Generally, two stage detection-estimation based filtering approaches are widely preferred in which the use of a separate noise detection scheme succeeded by a noise restoration scheme ensures that only the noisy pixels are filtered. This helps avoid the image blurring caused by the filtering of noise-free pixels. Filtering of only noisy pixels results into better preservation of detail features. Most of the impulse detection methods available in the literature require a decision threshold value [16], [17] and [21] to make an intelligent decision whether the pixel under consideration is noisy or not. 
Thus the selection of the decision threshold value impacts the performance of the impulse detector in the sense that, the number of false and m33issed detections is under its direct influence. Some of the recently reported two stage grey and color image denoising are high performance detection (HPD) [21], directional weighted median filter (DWM) [22], and switching vector median filter based on CIELAB color space [23].

For color image denoising component-wise filtering and the vector processing approach [24] are popularly used. The main drawback of applying the component-wise approach for filtering is that the inherent correlation existing among pixels of different channels may be lost, resulting into the generation of color artifacts in the filtered images. However the noise removal ability of component-wise filtering approaches is quite appreciable. On the other hand vector processing schemes aim at noise suppression without introducing the color artifacts, but fail to yield the reduced mean square error (MSE). Practical Color image denoising applications choose either of these approaches depending on the requirements. Most commonly used vector filters for noise removal in color images are the Vector Directional filter (VDF) [24] and the Directional Distance Filter (DDF) [25], Vector Median Filter (VMF) and those reported in [26], [27], [28] and [29].

It is also observed that the use of fixed value of threshold is not the ultimate solution for denoising of different types of images and ideally it should be changed adaptively according to the contents of the filtering window [9], [15], [16], and [18].

In this paper, we present an impulse detection method where the decision threshold is dynamic and adaptively changes on the basis of pixel statistics within the filtering window. This is followed by a simple filtering method to replace the noisy pixels. The proposed method entitled "A Novel Adaptive Tolerance Filter (ATF) for Denoising the Digital Grey and Color Images" uses an adaptive tolerance for noise detection and a simple filtering scheme for the restoration of noisy pixels and aims to yield much better quantitative parameters such as Peak Signal to Noise Ratio (PSNR), Mean Square Error (MSE), Structural Similarity Index (SSIM) than the other state of art denoising methods for grey and color image denoising.

\section{Noise model}

Two common classes of impulsive noise that interfere with the images during acquisition, transit, storage and retrieval are salt and-pepper noise (SPN) and random-valued impulse noise (RVIN). Our work presented in this paper addresses the suppression of the RVIN with details preserved intact to a larger extent. The RVIN noise model is described as follows:

\subsection{Random valued impulsive noise (RVIN)}

A digital image $\mathrm{X}_{\mathrm{RVIN}}(\mathrm{i}, \mathrm{j})$ contaminated with RVIN of density ' $\mathrm{d}$ ' is mathematically represented as in equation (1) and is represented in fig (1).

$X_{\text {RVIN }}(i, j)=\left\{\begin{array}{c}X(i, j) \text { with probabilty } \quad p=1-d \\ \eta(i, j) \text { with probabilty } p=d\end{array}\right.$

In the above equation $\eta(\mathrm{i}, \mathrm{j})$ represents a uniformly distributed random variable, ranging from ' 0 ' to ' 255 ', that replaces the original pixel value $\mathrm{X}(\mathrm{i}, \mathrm{j})$. The magnitude of noise at any noisy pixel location $(\mathrm{i}, \mathrm{j})$ is uniformly distributed between the minimal (0) and maximal (255) possible pixel values as in equation (1) and are independent of the original pixel magnitude.

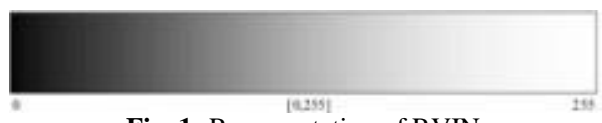

Fig. 1: Representation of RVIN.

\section{Proposed work}

In this section details of the proposed work entitled "Adaptive Tolerance based Filter (ATF) for Noise Suppression in Digital Grey and Color Images" is presented.

In general, it is observed that a noisy pixel takes intensity value which is explicitly different from the neighborhood pixels in the working window in the noise affected regions as compared to the noise-free regions in the image that have smoothly varying pixel luminance values separated by edges. The proposed impulse detection strategy exploits this fact. Details of the impulse noise detection are outlined below: 


\subsection{Outline of the proposed work}

a) Define $n \times n$ filtering window $W_{n \times n}$ with center pixel $X_{i, j}$ ( in color images $W_{n \times n}(K)$ for channel ' $K$ ' with centre pixel $\left.\mathrm{X}_{\mathrm{i}, \mathrm{j}}(\mathrm{K})\right)$, where $\mathrm{K}=1,2,3$.

b) Let $X_{i, j}(k)=\left\{X_{i, j}(1), X_{i, j}(2) X_{i, j}(3)\right\}$ be the three vector channel pixels in the RGB space at location (i,j) of random valued impulse noise affected image ' $\mathrm{X}$ '.

c) Firstly, the mean (or average) of already processed pixels in the current working window (of size $\mathrm{n} \times n$ ) say, $\mu_{\mathrm{P}}$ $\left(\mathrm{W}_{\mathrm{n} \times \mathrm{n}}\right),\left(\right.$ or $\mu_{\mathrm{P}}\left(\mathrm{W}_{\mathrm{n} \times \mathrm{n}}(\mathrm{K})\right.$ in color image) is computed.

d) A new trimmed vector of window pixels $\left\{X_{t}\right\}$, (or $\left\{X_{t}^{(\mathrm{K})}\right\}$ in color image) is obtained from input vector of Pixels $\left\{\mathrm{X}_{\mathrm{t}}\right\}$ (or $\left\{\mathrm{X}_{\mathrm{t}}(\mathrm{K})\right\}$ in case of color images).

e) Then the noisy pixels are replaced by their estimates.

f) Impulse detection and filtration are performed for each channel separately in case of color images.

\subsection{Description of the proposed work}

Filter output in general is based upon the type of operation performed on the group of pixels within a working sliding window. The outputs of average and median filters on ' $N$ ' number of pixels within a window are different. Each operation results with some merits and demerits and thus the average and median filters complement each other. Thus the main objective of the proposed work is to develop and implement an adaptive tolerance based impulse detection scheme and a filter based on the arithmetic averaging (which is free from sorting operation as in median filters). Recursive version of this filter (after two to three iterations) yields an output same as the median filter output.

\subsubsection{Impulse detection}

Proposed filter chooses a filter of an appropriate size of the window as will be explained in section 4.1. To illustrate the details of the impulse detection a sample working window of size $3 \times 3$ is shown in figure 3 (a). Various steps involved in the proposed impulse detection are explained as below:

Step 1) Compute the arithmetic mean of processed pixels $\left[\mu_{\mathrm{n} \times \mathrm{n}}\right]_{\mathrm{X}}$, (or $\left[\mu_{\mathrm{n} \times \mathrm{n}}{ }^{(\mathrm{K})}\right]_{\mathrm{X}}$, where $\mathrm{K}=1,2,3$ in case of color image ) belonging to the current working window as depicted in figure (2).

$\left[\mu_{\mathrm{n} \times \mathrm{n}}\right]_{\mathrm{X}}=\mu_{\mathrm{P}}=\frac{X 1+X 2+X 3+X 4}{4}$

\begin{tabular}{|l|l|l|}
\hline $\mathrm{X}_{1}$ & $\mathrm{X}_{2}$ & $\mathrm{X}_{3}$ \\
\hline $\mathrm{X}_{4}$ & $\mathrm{X}_{5}$ & $\mathrm{X}_{6}$ \\
\hline $\mathrm{X}_{7}$ & $\mathrm{X}_{8}$ & $\mathrm{X}_{9}$ \\
\hline
\end{tabular}

Fig. 2: A $3 \times 3$ working window with ' $X_{5}$ ' as the reference (or test) pixel. $X_{1}, X_{2}, X_{3}$ and $X_{4}$ are the processed pixels and $X_{6}, X_{7}, X_{8}, X_{9}$ are the pixels yet to be processed.

Step 3) Obtain a new trimmed vector of pixels from the vector ' $\mathrm{X}_{\mathrm{i}}$ ' ( or ' $\mathrm{X}_{\mathrm{i}}^{(\mathrm{K})}$, in color images) as $\left\{\mathrm{X}_{\mathrm{t}}\right\}$, or $\left(\mathrm{X}_{\mathrm{t}}^{(\mathrm{K})}\right.$ in color image), where

$\mathrm{X}_{\mathrm{t}}=\left\{\begin{array}{l}\mu p+\mathrm{Td} ; \text { for all } \mathrm{Xi}>\mu \mathrm{p}+\mathrm{Td} \\ \mu p-\mathrm{Td} ; \text { for all } \mathrm{Xi}<\mu \mathrm{p}-\mathrm{Td}\end{array}\right.$

$\mathrm{X}_{\mathrm{t}}^{(\mathrm{K})}=\left\{\begin{array}{cc}\mu p+\mathrm{Td} ; & \text { for all } \mathrm{Xi}(\mathrm{K})>\mu \mathrm{p}+\mathrm{Td} \\ \mu p-\mathrm{Td} ; & \text { for all } \mathrm{Xi}(\mathrm{K})<\mu \mathrm{p}-\mathrm{Td}\end{array}\right.$

where $\mathrm{K}=1,2,3$ for color images.

In the above equation ' $T_{d}$ ' is the dynamic tolerance and ' $X_{t}{ }^{(k)}$ ' is array of pixels within the working window in each of the channels.

\subsubsection{Noise filtering}

The Trimmed Average Filter output ' $\mathrm{O}_{\mathrm{t}}$ ' is given by: $\mathrm{O}_{\mathrm{t}}=$ Average $\left(\mathrm{X}_{\mathrm{t}}\right)$

or

$\mathrm{O}_{\mathrm{t}}=$ Average $\left(\mathrm{X}_{\mathrm{t}}^{(\mathrm{K})}\right)$ for color images 


\section{Selection of parameters}

Generally the performance of the denoising filter is influenced by the filtering window size, decision tolerance etc. In several practical applications, selections of these parameters depend upon the amount of noise density and are quite specific for each type of the image. We discuss in detail the selections of these parameters as below:

\subsection{Adaptive window size}

With smaller size working window, the detail preservation is good, but noise elimination capability is weak; whereas with the bigger window size noise elimination ability is good at the cost of loss of detail preservation. Adaptive window serves the need of addressing the images corrupted with the low-to-heavy impulsive noise density. Thus a variable window size is essential to effectively suppress the corrupted pixels while preserving the fine details of an image.

By considering and unifying the goodness of small and large working windows, in our work it is possible to adaptively determine the size of the filtering windows according to the amount of interfering noise density. Initially, we start with a $3 \times 3$ working window and try to obtain the filtering performance in terms of Mean Square Error (MSE) and Peak Signal to Noise Ratio (PSNR) on the standard test images (say 'Lena', 'Baboon' etc) with different noise densities. Based on the results obtained, we suggest different window sizes for a particular range of noise densities as presented in table (1).

Table 1: Suggested Size of the Working Window for Impulsive Noise Density 'd' (Estimated)

\begin{tabular}{cc}
\multicolumn{2}{c}{ Table 1: Suggested Size of the Working Window for Impulsive Noise Density 'd' (Estimated) } \\
\hline \% Noise Density & Window size (Suggested) \\
\hline $0-40 \%$ & $3 \times 3$ \\
$50-70 \%$ & $5 \times 5$ \\
$80-90 \%$ & $7 \times 7$ \\
\hline
\end{tabular}

\subsection{Problems with fixed tolerance and computation of dynamic tolerance}

It is generally observed that any tolerance based switching filter that makes use of a fixed noise-detection tolerance obtained at a particular value of noise density suffer from the lack of adaptivity to the changes in noise density. Thus a mismatch between the designed algorithms and the actual amount of noise density (which is often not known) will cause a significant and noticeable changes and a substantial degradation on the performance of filters. Moreover, with the increase in noise density, the percentage misclassifications increase and finally results in poor filtering performance. Therefore, an intelligent noise-detection process will be highly desirable, appreciable and instrumental in correctly identifying different types of pixel characteristic. In addition, an adaptive filtering scheme is essential to effectively remove the corrupted pixels while preserving image details when misclassification of pixel characteristic occurs. These requirements indicate that both noise detection and the corresponding filtering operation are crucial to achieve good filtering performance, especially at high noise density interference.

\subsubsection{Dynamic tolerance selection}

Dynamic or adaptive tolerance value plays an important role in differentiating the edge of the image and the noisy pixel [3], [5]. Thus the most critic technique to achieve the goal of noise suppression with detail preservation is to find a proper dynamic truncation threshold in 2-stage detection-estimation based switching filters. Steps involved in computing the adaptive decision tolerance are as follows:

Step 1) For the chosen working window of size $\mathrm{W} \times \mathrm{W}$, compute the average of processed pixels ' $\mu_{\mathrm{P}}$ ' as already explained in section 3.1.

Step 3) Now compute an adaptive (or dynamic) tolerance ' $T_{D}$ ' for the use of decision making in impulse detection stage as follows :

$\mathrm{T}_{\mathrm{D}}=\frac{1}{n} \sum_{i=1}^{n}\left(\left|\mathrm{X}_{\mathrm{i}}-\mu_{\mathrm{P}}\right|\right)$

\section{Noise removal in color images}

Noise filtering techniques in color images can be divided into two classes [29] as Component-wise methods and Vector methods. In component wise methods, the three primary color channels R, G and B are considered to be independent for filtering of noise and later they are combined to generate the filtered version of the color image. All the methods reported for the monochrome images can be used for noise removal in color images under this category. However this approach of color denoising suffers from the problem that the component filtering can generate color artifacts [27], [28]. On the other hand in vector methods, every color pixel is assumed as a vector and the faulty pixel is replaced by a noise free vector value within the filtering window. This approach is free from color artifacts as there are no new colors 
introduced into the image. The main principles of commonly used vector filters are as below:

The filtering algorithm must restore both the intensity and the color of the image effectively. This objective can be fulfilled with the efficient use of information available in three channels of the noise-free components and preferably, the filtering algorithm should attempt to change only the noisy component in order to assure that the restored pixel is nearer to the original one [29].However the vector approaches of denoising schemes fail to provide the required performance in terms of PSNR and RMSE measures.

In our work, the scalar median filtering approach has been used, i.e. in the RGB color space used in our work, each pixel at the location (i,j) can be represented as color vector $\mathrm{O}_{i, j}=\left[\mathrm{O}_{\mathrm{i}, \mathrm{j}}{ }^{\mathrm{R}} \mathrm{O}_{\mathrm{i}, \mathrm{j}}{ }_{\mathrm{G}}^{\mathrm{G}} \mathrm{O}_{\mathrm{i}, \mathrm{j}}{ }^{\mathrm{B}}\right]$, where $\mathrm{O}_{\mathrm{i}, \mathrm{j}}{ }^{\mathrm{R}}, \mathrm{O}_{\mathrm{i}, \mathrm{j}}{ }^{\mathrm{G}}$, and $\mathrm{O}_{\mathrm{i}, \mathrm{j}}{ }^{\mathrm{B}}$ are the Red (R), Green (G), and Blue (B) components respectively. To model the noisy color images RVIN of a particular density is injected to each of these color components which in turn implies that when a color component is being noised by noise density of $20 \%$, it means that each color component is being corrupted by a noise density of $20 \%$. Thus, for each pixel $\mathrm{O}_{\mathrm{i}, \mathrm{j}}$, the corresponding pixel of polluted image will be denoted as $X_{i, j}=\left[X_{i, j}{ }^{R}, X_{i, j}{ }^{G}, X_{i . j}{ }^{B}\right]$ in which the probability density functions of each color components are the same.

\section{Results and discussion}

Performance of the proposed ATAF scheme is validated with the MATLAB simulation performed on synthetic images like Cameraman, Lena, Baboon, Pepper etc. noised with random valued impulsive noise. At various noise densities the size of the window is made variable as suggested in Table (1).Qualitative as well as quantitative results are obtained and the evaluations have been made on several restored images. The objective measures used are:

1) Peak Signal to Noise Ratio (PSNR)

PSNR $=10 \log _{10} \frac{255^{2}}{\mathrm{MSE}}$

2) Mean Square Error (MSE) for an image of size $\mathrm{M} \times \mathrm{N}$ is defined as

$\operatorname{MSE}=\frac{1}{\operatorname{MXN}} \sum_{\mathrm{i}=0}^{\mathrm{M}-1} \sum_{\mathrm{j}=0}^{\mathrm{N}-1}[\mathrm{I}(\mathrm{i}, \mathrm{j})-\mathrm{O}(\mathrm{i}, \mathrm{j})]^{2}$

where $I[i, j]$ is the noisy image and $\mathrm{O}[\mathrm{i}, \mathrm{j}]$ is the denoised image.

3) Structural Similarity (SSIM) Index: The SSIM Index is calculated on various windows of an image. The measure between two windows ' $\mathrm{X}$ ' and ' $\mathrm{Y}$ ' of common size $\mathrm{n} \times \mathrm{n}$ is:

$$
\text { SSIM Index }=\left[\left(2 \mu_{\mathrm{x}} \mu_{\mathrm{y}}+\mathrm{C}_{1}\right)\left(2 \Omega_{\mathrm{xy}}+\mathrm{C}_{2}\right)\right] /\left[\left(\mu_{\mathrm{x}}\right)^{2}+\left(\mu_{\mathrm{y}}\right)^{2}+\mathrm{C}_{1}\right]\left[\left(\Omega_{\mathrm{x}}\right)^{2}+\left(\Omega_{\mathrm{y}}\right)^{2}+\mathrm{C}_{2}\right]
$$

where $\mu_{x}=$ Average of ' $x$ ', $\mu_{y}=$ Variance of ' $y$ ', $\Omega_{x}{ }^{2}=$ Variance of ' $x$ ', $\Omega_{y}{ }^{2}=$ Variance of ' $y$ ', $\Omega_{x y}=C o$-variance of ' $x$ ' and ' $y$ ', $\mathrm{C}_{1}=\left(\mathrm{K}_{1} \mathrm{~L}\right)^{2}$ and $\mathrm{C}_{2}=\left(\mathrm{K}_{2} \mathrm{~L}\right)^{2}$ are the variables used to stabilize the divisor with weak denominator, ' $\mathrm{L}$ 'is the Dynamic range of the pixel values, $\mathrm{K} 1=0.01$ and $\mathrm{K}_{2}=0.03$ by default.

As said earlier, the effective use of this method requires optimum values for parameters such as maximum window size ' $\mathrm{W}_{\max }$ ', and the dynamic threshold values $\mathrm{T}_{\mathrm{d}}$. To maximize the probabilities that only wanted pixels take part in filtering operation and to reduce the computational time, above said parameters must be chosen of appropriate values.

Table 2 presents the Peak Signal to Noise Ratio (PSNR) and Root Mean Square Error (RMSE) results comparison of proposed novel Adaptive Tolerance Filter (ATF) with various other filters namely SMF, WMF, UTMF and TMF and Ref (12) obtained for the noisy 'Lena grey' image contaminated with random valued salt and pepper noise of densities ranging from $10 \%$ to $90 \%$. From the results it is evident that the proposed filter out performs all other filtering schemes that use fixed and empirically obtained threshold values and fixed size windows. However the simulation results of UTMF and Ref (12) filters are somewhat encouraging as compared to SMF, WMF and TMF. From the comparative analysis we can conclude that the filters designed for salt and pepper impulse noise suppression, exhibit poor performance in suppressing the random valued impulse noise. Figures 2 and 3 present the PSNR and RMSE results tabulated in table (1) in the form of bar chart.

Table 3 presents the PSNR and MSE results comparison of proposed filter at different RVIN for Baboon color and Lena grey images.

Table 4 summarizes the results of Structural Similarity (SSIM) Index comparison results obtained for Baboon and Lena color images. From these results it is evident that the goodness of the denoising filter depends upon the nature and the structural contents of the image. Since Baboon image is structurally complex than the Lena image, the SSIM results of Baboon are less encouraging as compared to the results obtained for Lena image. Results tabulated in 4 are represented in the form of bar graph for the purpose of ease of analysis.

Table 5 presents the performance comparison of the results obtained with the proposed filter on standard color images, Lena and Baboon noised with 50\% RVIN in terms of PSNR, MSE and SSIM parameters. Results shown in figures (5) and (6) are obtained with the proposed filter for the Baboon and Lena grey images corrupted with 50\% RVIN. From the visual analysis it is evident that the proposed filter performs better compared to several other states of art filters in 
suppressing the random valued impulsive noise to a greater extent. From the results it can be concluded that the denoising performance of the proposed filter is good in the RVIN environment.

Set of figures in (7) are obtained for the Lena grey image noised with 50\% RVIN. From the visual analysis we can conclude that the denoising performance results of the proposed NAF is much superior to the SMF,TMF,UTMF,WMF and the filter proposed in Ref [12].

Set of figures in (8) are 50\% 30\%, 80\% and 90\% RVIN affected Lena color images. The corresponding denoised results of the proposed filter are shown in set of figures (9). From these perceptual results it can be concluded that the performance of the proposed filter is good with respect to color image denoising with a little sacrifice for the details such as the edges.

Table 2: PSNR and MSE Results Comparison of Various Filters for Lena(Grey) Image Noised with RVIN

\begin{tabular}{|c|c|c|c|c|c|c|c|c|c|c|}
\hline \multirow{2}{*}{$\begin{array}{l}\text { Filter } \\
\% \text { ND }\end{array}$} & \multicolumn{5}{|c|}{ Peak Signal to Noise Ratio (PSNR) } & \multicolumn{5}{|c|}{ Root Mean Square Error (RMSE) } \\
\hline & $20 \%$ & $40 \%$ & $60 \%$ & $80 \%$ & $90 \%$ & $20 \%$ & $40 \%$ & $60 \%$ & $80 \%$ & $90 \%$ \\
\hline SMF & 29.62 & 19.03 & 12.44 & 8.90 & 6.69 & 46.10 & 305 & 1330 & 3464 & 4883 \\
\hline WMF & 27.13 & 17.58 & 11.64 & 7.91 & 6.54 & 20.34 & 179 & 895 & 3672 & 5031 \\
\hline UTMF & 35.7 & 31.7 & 28.1 & 23.2 & 19.2 & 17 & 43 & 98 & 310 & 766 \\
\hline $\mathrm{TMF}$ & 28.01 & 19.65 & 12.21 & 9.21 & 8.15 & 49.9 & 319 & 1712 & 3909 & 6098 \\
\hline Ref. 10 & 33.25 & 28.81 & 25.04 & 20.6 & 18.9 & 30.95 & 86.1 & 204.9 & 565.6 & 830 \\
\hline PA & 41.62 & 36.03 & 33.76 & 29.5 & 28.0 & 19.25 & 79.9 & 186.3 & 504 & 783 \\
\hline
\end{tabular}

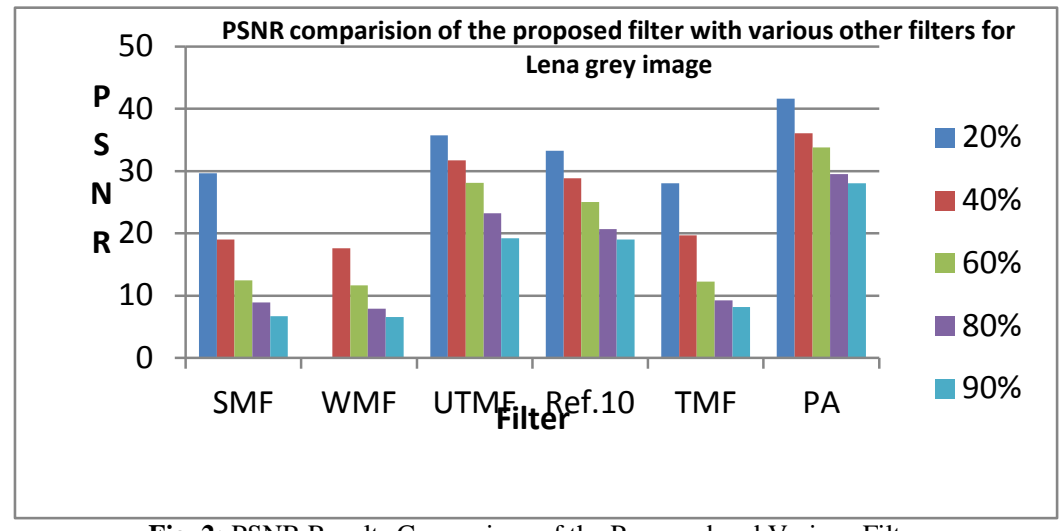

Fig. 2: PSNR Results Comparison of the Proposed and Various Filter

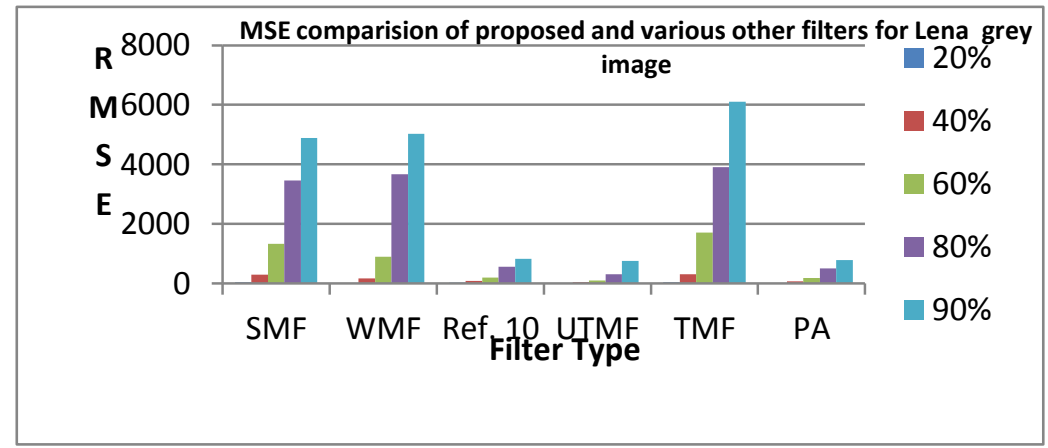

Fig. 3: RMSE Comparison Results of Proposed and Various other Filters.

Table 3: PSNR and MSE Performance Comparison of Proposed Filter at Different RVIN for Baboon Color and Lena Grey Images

\begin{tabular}{|c|c|c|c|c|}
\hline \multirow{2}{*}{$\%$ Noise Density } & \multicolumn{2}{|c|}{ Lena grey image with RVIN } & \multicolumn{2}{|c|}{ Baboon color image with RVIN } \\
\hline & PSNR & MSE & PSNR & MSE \\
\hline 20 & 41.62 & 19.25 & 36.54 & 32.6 \\
\hline 40 & 36.03 & 79.97 & 31.12 & 253.5 \\
\hline 60 & 33.76 & 186.3 & 28.54 & 792.4 \\
\hline 80 & 29.52 & 504.5 & 24.37 & 1103 \\
\hline 90 & 28.03 & 783.8 & 20.12 & 1756 \\
\hline
\end{tabular}


Table 4: SSIM Index Performance Comparison of Proposed Filter at Different RVIN For Baboon and Lena Color Images.

\begin{tabular}{ccc}
\hline$\%$ ND & Lena (Color) & Baboon(Color) \\
\hline 20 & 0.9002 & 0.7876 \\
40 & 0.7361 & 0.6043 \\
60 & 0.5003 & 0.4702 \\
70 & 0.4988 & 0.4024 \\
80 & 0.4815 & 0.3276 \\
90 & 0.4313 & 0.3197 \\
\hline
\end{tabular}

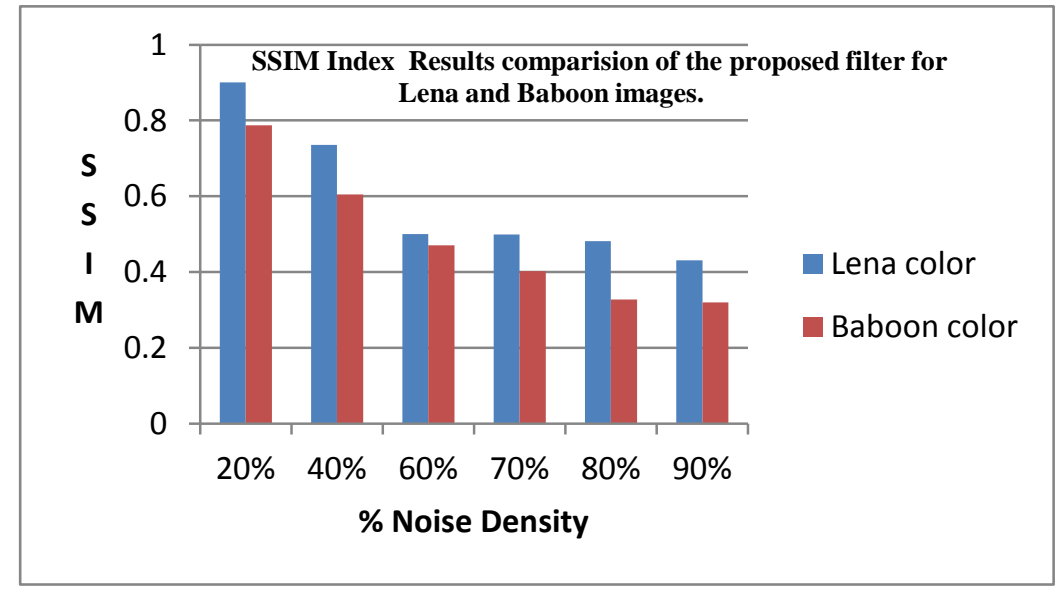

Fig. 4: SSIM Results Comparison of Lena and Baboon Color Images of the Proposed Scheme

Table 5: Performance Comparison of Proposed Filter for Lena and Baboon Color Images at 90\% RVIN

\begin{tabular}{lcc} 
& Table 5: Performance Comparison of Proposed Filter for Lena and Baboon Color Images at 90\% RVIN \\
\hline Parameter & Lena Image & Baboon Image \\
\hline PSNR & 27.28 & 20.12 \\
MSE & 915 & 1756 \\
SSIM & 0.4313 & 0.3197 \\
\hline
\end{tabular}

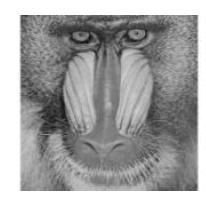

a)Original

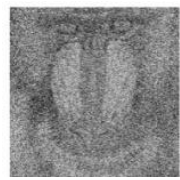

b)Noised

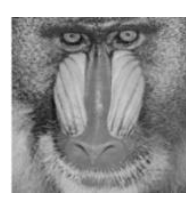

c)Denoised

Fig. 5: Results Obtained for 'Baboon' Image Corrupted with 50\% Random Valued Impulse Noise (RVIN) Density.

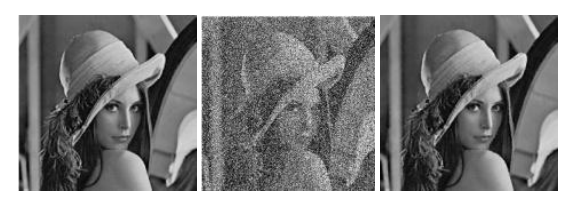
a)Original
b)Noised
c)Denoised

Fig. 6: Results Obtained for Lena Image Corrupted with 50\% Random Valued Impulse Noise (RVIN) Density. 


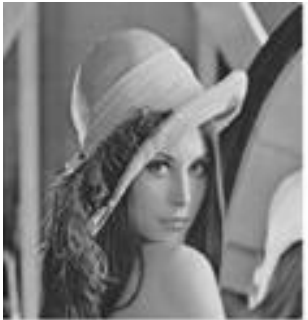

a)Original

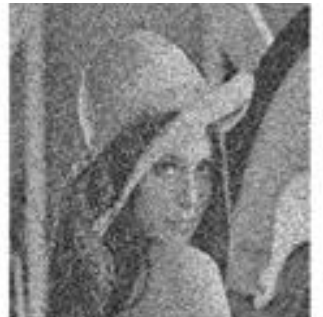

b)Noised

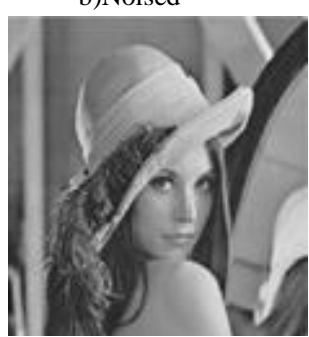

f)UTMF

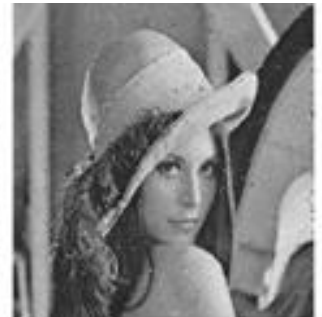

c)SMF

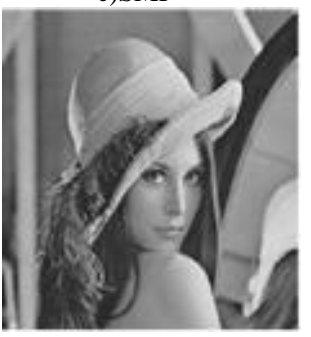

g)Ref.12

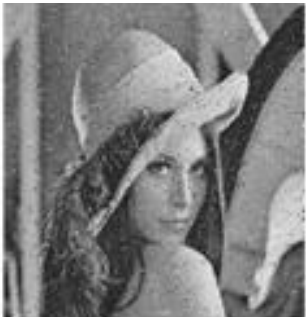

d)WMF

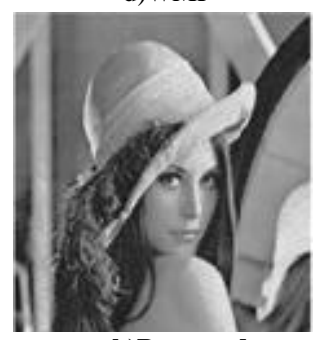

h)Proposed

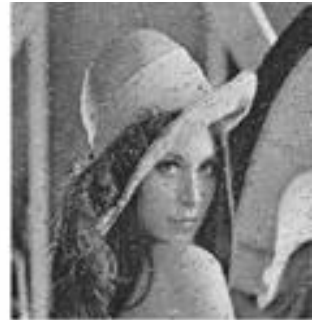

e)TMF

Fig. 7: Denoising Performance of Various Filters on 50\% RVIN added Lena Grey Image.
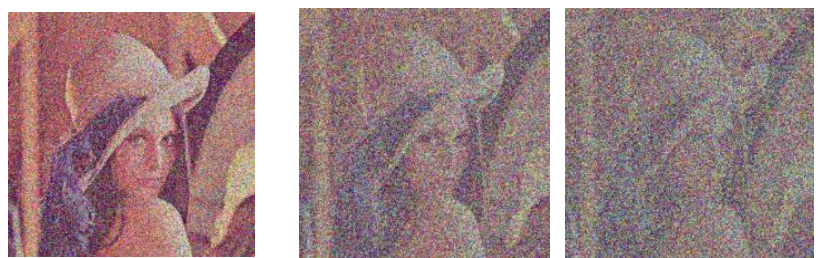

Fig. 8: Lena Color Image Noised with
a) $30 \% \mathrm{RVIN}$
b) $80 \%$ RVIN
c) $90 \%$ RVIN
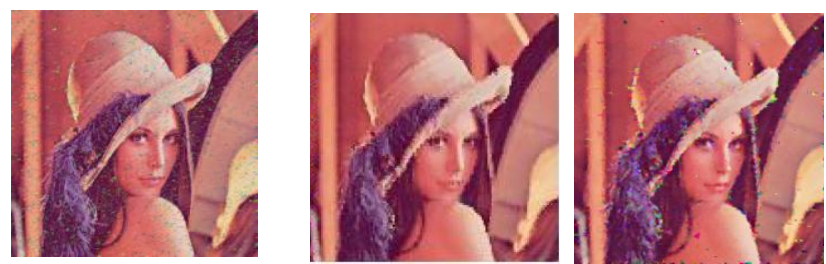

Fig. 9: Corresponding Denoised Images with the Proposed Filter

\section{Conclusion and future scope}

An efficient algorithm that suppresses the random valued impulsive noise (RVIN), from the heavily damaged digital a grey and color image without adding much edge distortions is presented in this paper. Proposed Adaptive Tolerance Filter (ATF) scheme is flexible in the sense that, it uses adaptive tolerance and adaptive window size in the noise detection stage. Decision tolerance and the size of the filtering windows are based on the intensity of interfering noise. Its performance in suppressing RVIN from the grey images is much encouraging as compared to the RVIN suppression from the color images in terms of PSNR, MSE and the perceptual results. However the overall performance of the proposed filter is much better in comparison with the several other competitive filter algorithms proposed for this purpose. From the simulation results it is evident that the proposed filter scheme can be a very good choice for random valued impulsive noise (RVIN) suppression with detail preservation in noisy grey and color images noised up to 70\% very effectively, whereas its performance reduces at noise densities more than $70 \%$. This issue can be addressed in future work. The proposed filter scheme is designed for 8 bit still images. The method can be enhanced for noisy image sequences, and super resolution images.

\section{Acknowledgements}

Authors express their deep gratitude towards the peer reviewers for providing feedbacks and valuable suggestions to improve the quality of the manuscript. 


\section{References}

[1] R.C.Gonzalez, R.E. Woods, "Digital Image Processing (2nd Edition)", Prentice Hall/ Addison-Wesley; 2002.

[2] Sonali R. Mahalale, Nileshsingh V.T., "A Comparative Study of Image Filtering on Various Noisy pixels", International Journal of Image Processing and Vision Sciences, ISSN (Print): 2278 - 1110, Volume-1, Issue-2, 2012.

[3] Sin Hoong Teoh, Boon Tatt Koik, and Haidi Ibrahim, "Exploration of Current Trend on Median Filtering Methods Utilized in Digital Grayscale Image Processing", International Journal of Materials, Mechanics and Manufacturing, Vol. 1, No. 1, February 2013.

[4] Ajay Kumar Nain, Surbhi Singhania, Shailender Gupta, Bharat Bhushan, "A Comparative Study of Mixed Noise Removal Techniques", International Journal of Signal Processing, Image Processing and Pattern Recognition,Vol.7,No.(2014),pp.405-414, http:// dx.doi.org/10.14257/ijsip.2014.7.1.37 ISSN: 2005.

[5] Pragati Agarwal,Jayendra Singh Verma, "A Survey of Linear and Non-Linear Filters for Noise Reduction ",International Journal of Advance Research in Computer Science and Management Studies", ISSN: 2321-7782,Vol.1, Issue 3, August,2012.

[6] Z.Wang, D. Zhang, "Progressive Switching Median Filter for the Removal of Impulse Noise from Highly Corrupted Images, "IEEE Transactions on Circuits and Systems II: Analog and Digital Signal Processing, Vol. 46, no. 1, pp. 78-80, 1990. http://dx.doi.org/10.1109/82.749102.

[7] Madhu S. Nair, K. Revathy, Rao Tatavarti, Removal of Salt-and Pepper Noise in Images: A New Decision-Based Algorithm, Proceedings of the International Multi Conference of Engineers and Computer Scientists, 2008 Vol II, MECS 2008, 19-21 March, 2008.

[8] S.Esakkirajan, T.Veerakumar, Adabala, N.Subramanyam and C.H. Prem Chand, "Removal of High Density Salt and Pepper Noise Through Modified Decision Based Unsymmetrical Trimmed Median Filter," IEEE Signal Processing Letters, Vol. 18, No.5, May 2011. http://dx.doi.org/10.1109/LSP.2011.2122333.

[9] Mahdi Shaneh, and Arash Golibagh Mahyari, "Image Enhancement using $\alpha$-Trimmed Mean $\varepsilon^{-}$Filters, World Academy of Science, Engineering and Technology, Vol:5 2011-11-22.

[10] 9Xudong Jiang,"Iterative Truncated Arithmetic Mean Filter and Its Properties", IEEE Transactions on Image Processing, Vol.21, No.4, April 2012.

[11] K.Vasant, S.Kartik,"Performance Analysis Of Unsymmetrical Trimmed Median As Detector On Image Noise And Its FPGA Implementation",International Journal Information Sciences And Techniques (IJIST),Vol.2.No.3, May 2012,DOI:10.5121/ijist.2012.2302. http://dx.doi.org/10.5121/ijist.2012.2302.

[12] A.Rajmani,V.Krishnaveni, "Impulse Denoising Algorithm for Grey and RBG Images", International Journal of Computer Applications (0975 - 8887) Volume 70- No.2, May 2013.

[13] Srinivasan, K.S., Ebenezer. D.,"A New Fast and Efficient Decision Based Algorithm for Removal of High-density Impulse Noise," IEEE Signal Processing Letters, Vol.14, no. 3, pp.189-192, 2007. http://dx.doi.org/10.1109/LSP.2006.884018.

[14] A. Fabijanska and D. Sankowski, "Noise adaptive switching median-based filter for impulse noise removal from extremely corrupted images," IET Image Processing, Vol. 5, issue 5, pp. 472-480, 2011. [15] Geeta Hanji and M.V.Latte, "Detail Preserving Fast Median Based Filter," Journal of Advanced Computer Science and Technology, 1(4) (2012) 195-206 (C) Science Publishing Corporation, www.sciencepubco.com/index.php/JACSTarticle/view/24.

[15] Geeta Hanji and M.V.Latte,"A New Threshold Based Median Filtering Technique for Salt and Pepper Noise Removal," 2ndInternational Conference on Digital Image Processing-(ICDIP-2010), 2010 Proceedings of SPIE, Vol.7546, PP754639-754639-10, SINGAPUR, 2010, DOI: $10.1117 / 12.856333$. http://dx.doi.org/10.1117/12.856333.

[16] Geeta Hanji, M.V.Latte, "Novel Adaptive Filter for Impulse Noise Suppression from Digital images", International Journal Of Bioinformatics \& Biosciences (IJBB), Vol.4, No.3/4, December 2014.

[17] Geeta Hanji, M.V.Latte, "A New Impulse Noise Detection and Filtering Algorithm," Image Processing \& Communications (IPC), The Journal of University of Technology and Life Sciences in Bydgoszcz, Vol. 16, no.1-2, pp.43-48, DOI: 10.2478/v10248-012-0004-4. http://dx.doi.org/10.2478/v10248-012-0004-4.

[18] Geeta Hanji, M.V.Latte, "Reliable Filters for Impulse Noise Suppression", and International Journal of Soft Computing and Engineering (IJSCE), Blue Eyes Intelligence Engineering \& Sciences Publication Pvt. Ltd, ISSN: 2231-2307, Volume-4 Issue-6, January 2015.

[19] A. Rajamani, V. Krishnaveni, "A New Denoising Approach for the Removal of Impulse Noise from Color Images and Video Sequences", Image Anal Stereol 2012;31:185-191 doi:10.5566/ias.v31.p185-19.1.

[20] Awad AS, Man H.,"High Performance Detection filter for Impulse Noise Removal in Images," Electronic Letters, 2008 ; 3(44):192-4. http://dx.doi.org/10.1049/el:20083401.

[21] Dong Y, Xu S., "A new Directional Weighted Median Filter for Removal of Random-Valued Impulse Noise", IEEE Signal Processing Letters, 2007; 14(3): 193-6. http://dx.doi.org/10.1109/LSP.2006.884014.

[22] Jin L, Li D. "A switching Vector Median Filter based on the CIELAB Color Space for Color Image Restoration," Signal Processing, 2007; 87(6):1345-54. http://dx.doi.org/10.1016/j.sigpro.2006.11.008.

[23] Trahanias P.E, Venetsanopoulos A.N., "Vector Directional Filters -A New class of Multichannel Image Processing Filters," IEEE Transactions on Image Processing, 1993; 2(4):528-34. http://dx.doi.org/10.1109/83.242362.

[24] Karakos DG, Trahanias PE.,Generalized Multichannel Image-Filtering structures, IEEE Transactions On Image Processing, 1997;6(7): 103845 http://dx.doi.org/10.1109/83.597278.

[25] Astola J, Haavisto P, Neuov Y, "Vector Median Filters," Proceedings of IEEE, 1990; 78(4):678-89. http://dx.doi.org/10.1109/5.54807.

[26] Dang D, Luo W., "Color image noise removal algorithm utilizing hybrid vector filtering", International Journal of Electronic Communication, 2008;62(1):63-7. http://dx.doi.org/10.1016/j.aeue.2007.02.001.

[27] M. Emre Celebi, Hassan A. Kingravi, Y. Alp Aslandogan, "Nonlinear Vector Filtering for Impulsive Noise Removal From Color Images", Journal of Electronic Imaging, 16(3): 033008, 2007. http://dx.doi.org/10.1117/1.2772639.

[28] J. Astola, P. Haavisto, Y. Neuovo, "Vector Median Filters", IEEE Proceedings, Vol. 78, pp. 678-689, 1990 .

[29] Borgan Smolka, Konstantionos Plataniotis, "Nonlinear Techniques for Color Image Processing ", Invited Chapter to appear in "Nonlinear Signal and Image Processing: Th9eory, Methods, and Applications", CRC Press. 